\title{
Special issue: engineering applications of neural networks
}

\author{
Lazaros Iliadis $^{1} \cdot$ Chrisina Jane $^{2}$
}

Published online: 22 October 2016

(C) The Natural Computing Applications Forum 2016

This special issue is related to engineering applications of neural networks (EANN). Initially, the Program chairs of the 16th EANN 2016 conference selected a series of quality papers that were accepted and presented orally. Their authors were asked to submit extended versions (at least by $50 \%$ ) for potential inclusion in the Special Issue of the Neural Computing and Applications (NCA) scientific Journal. At the same time, several scientists from all around the globe submitted their manuscripts for consideration through an open process. Thus, the editors came up with a wide pool of very interesting papers. Totally 21 papers were submitted and passed through peer review by two or three independent academic referees. After a long and careful evaluation process, 13 were accepted, based on quality and novelty criteria.

The first paper is entitled "Hybrid Multistep Modeling for Calculation of Carbon Efficiency of Iron Ore Sintering Process Based on Yield Prediction," and it is authored by Xiaoxia Chen (Central South University, Changsha, China), Xin Chen (China University of Geosciences, Wuhan, China), Jinhua She (Tokyo University of Technology, Hachioji, Tokyo) and Min Wu (China University of Geosciences, Wuhan, China). It is related to a typical engineering (iron ore sintering powered by combustion of carbon) modeling approach. A hybrid multistep model was built to calculate the comprehensive carbon ratio (a measure of carbon efficiency). More specifically, the

Lazaros Iliadis

liliadis@fmenr.duth.gr

Chrisina Jane

chrisina.jayne@gmail.com

1 Democritus University of Thrace, Orestiada, Greece

2 Robert Gordon University, Aberdeen, Scotland, UK introduced algorithm uses fuzzy logic and a particle swarm optimization-based Artificial Neural Network.

Harris Papadopoulos, Efthyvoulos Kyriacou (Frederick University, Cyprus) and Andrew Nicolaides (Imperial College London, UK and Cyprus CDER Trust) are the authors of the second paper entitled: "Unbiased Confidence Measures for Stroke Risk Estimation based on Ultrasound Carotid Image Analysis." More specifically, they propose an approach for providing well-calibrated confidence measures for determining cerebrovascular risk stratification, based on characteristics from non-invasive ultrasound imaging of carotid plaques. They follow a modified version of the Conformal Prediction method, called Label-conditional Mondrian Conformal Prediction which is combined with an underbagging ensemble of Artificial Neural Networks so that its outputs are based on unbiased estimates.

The third paper "An RBF Neural Network Approach to Geometric Error Compensation with Displacement Measurements Only" is written by a group of scientists, namely Rui Yang (Shandong University of Science and Technology, Qingdao, China) Kok Kiong Tan, Arthur Tay, Sunan Huang, Jie Sun, Jerry Fuh, Yoke San Wong, Chek Sing Teo (National University of Singapore) and Zidong Wang (Shandong University of Science and Technology, Qingdao, China). It presents a novel radial basis function neural network, based on the geometric error compensation method with displacement measurements. The experimental testing has showed a high level of accuracy which is very inspiring.

Invasive species is a timely problem met all over the globe, due to climate change. A hybrid bio-inspired computational intelligence model, employing Extreme Learning Machines combined with an evolving Izhikevich spiking neuron model is developed and employed for the automated identification of the harmful invasive fish 
species "Lagocephalus sceleratus." The identification is based on the analysis of environmental DNA. Konstantinos Demertzis and Lazaros Iliadis (Democritus University of Thrace, Greece) have authored this contribution which seems to address this problem in an efficient manner.

David Lehký and Martina Šomodíková (Brno University of Technology, Czech Republic) present a paper entitled: "Reliability Calculation of Time-Consuming Problems. Using a Small-Sample Artificial Neural Network-Based Response Surface Method." An important step when designing and assessing the reliability of existing structures and/or structural elements in complex systems (e.g., bridges) is to calculate the reliability level described by failure probability or reliability index. This is a time-consuming task that requires the utilization of approximation methods with a view to reducing the computational effort to an acceptable level. This paper introduces an Artificial Neural Network as an approximation (a so-called response surface) of the original limit state function. In order to be as effective as possible with respect to computational effort, a stratified Latin hypercube sampling simulation method is utilized to properly select training set elements. Subsequently, the Artificial Neural Network-based response surface is utilized to calculate failure probability. To increase the accuracy of the determined failure probability, the response surface can be updated close to the failure region.

The sixth paper is authored by Robin Babujee Jerome and Kimmo Hätönen (Aalto University, Espoo, Finland). Its tile is the following: "Anomaly Detection and classification using a metric for determining the significance of failures. Case study: Mobile Network Management data from LTE network." Big data analytics and machine learning applications are often used to detect and classify anomalous behavior in telecom network measurement data. The accuracy of findings during the analysis phase greatly depends on the quality of the training data set. If the training data set contains data from Network Elements (NEs) with high number of failures and high failure rates, such behavior will be assumed as normal. There exists no universally accepted method for effective classification of anomalous behavior. Operators use well-known indicators such as, Drop Call ratio and Handover failure ratio to identify misbehaving NEs. This paper proposes a method of measuring the significance of failures. The usage of this method is proposed in two stages of Anomaly Detection: training set filtering (preprocessing stage) and classification of anomalies (post-processing stage) using an automated process.

The seventh paper is entitled "Training Echo State Networks for Rotation-Invariant Bone Marrow Cell Classification." The authors are: Philipp Kainz (University of Graz, Austria), Harald Burgsteiner (Institute for eHealth,
Graz University of Applied Sciences, Austria), Martin Asslaber (Institute of Pathology, Medical University of Graz, Austria) and Helmut Ahammer (Institute of Biophysics, Center for Physiological Medicine, Medical University of Graz, Austria). It is related to medical diagnostic application of Neural Networks. It is well known that a confident examination of bone marrow specimen is dependent on a valid classification of myeloid cells. In this work, a novel rotation invariant learning scheme is proposed for multi-class Echo State Networks (ESNs), which achieves very high performance in automated bone marrow cell classification. Based on representing static images as temporal sequence of rotations, this research shows how ESNs robustly recognize cells of arbitrary rotations by taking advantage of their short-term memory capacity. The performance of this approach is compared to a classification random forest $(\mathrm{RF})$ that learns rotation invariance in a conventional way by exhaustively training on multiple rotations of individual samples.

Ander Arriandiaga, Eva Portillo, Jose Antonio Sánchez, Itziar Cabanes and Asier Zubizarreta (University of the Basque Country, Spain) have written the eighth paper, entitled: "Recurrent ANN based modelling of the dynamic evolution of the Surface Roughness in Grinding." Grinding is critical in modern manufacturing due to its capacity for producing high surface quality and high precision parts. One of the most important parameters that indicate the grinding quality is the surface roughness. Several authors in the literature have employed ANN that predicts surface finish. However, all of these approaches provide a particular solution for a wheel-workpiece pair, not generalizing to new grinding wheels. Besides, these solutions do not give surface roughness values related to the grinding wheel status. This work describes a successful modeling of the dynamic evolution of the surface roughness, based on Recurrent Neural Networks with the capability to generalize to new grinding wheels and conditions taking into account the wheel wear. Results show good prediction of the surface finish dynamic evolution.

The ninth paper "Local Learning Regularization Networks for localized regression" is authored by Yiannis Kokkinos and Konstantinos Margaritis (University of Macedonia, Thessaloniki Greece). This paper considers local learning versions of Regularization Networks and investigates several options for improving their online prediction performance, both in terms of accuracy and speed. First this effort exploits the interplay between locally optimized and globally optimized hyper-parameters (regularization parameter and kernel width) where each new predictor needs to optimize online. By comparing Eigenvalue decomposition (EVD) with Cholesky decomposition specifically for the local learning training and testing phases, the authors reveal that the Cholesky-based 
implementations are faster than their EVD counterparts for all the training cases. While EVD is suitable for validating cost-effectively several regularization parameters, Cholesky should be preferred when validating several neighborhood sizes (the number of $k$-nearest neighbors) as well as when the local network operates online.

The tenth paper is authored by Ali AghaeiRad (University of Coimbra Polo II, Pinhal de Marrocos, Coimbra, Portugal), Ning Chen (Henan Polytechnic University, Jiaozuo, Henan, China) and Bernardete Ribeiro (University of Coimbra Polo II, Pinhal de Marrocos, Coimbra, Portugal). This paper is about a financial application of Neural Computing. Credit scoring and financial risk estimation plus monitoring are crucial in the accounting and finance domain. Numerous pattern recognition techniques including neural networks, decision trees and support vector machines, have been published in the literature, to classify the borrowers as good or bad credit risk. This paper presents a hybrid credit scoring model and illustrates how the unsupervised learning, based on SelfOrganizing Maps, can improve the discrimination capability of Feed forward Neural Networks (FFNN). Within the hybridization scheme, the knowledge (i.e., prototypes of clusters) found by the SOM is transferred as input to the subsequent FNN model. The model is validated successfully with four real-world data sets.

Varun Kumar Ojha (VSB Technical University of Ostrava, Czech Republic and Jadavpur University, Kolkata, India) Parmartha Dutta (Visva-Bharati University, India) and Atal Chaudhuri (Jadavpur University, Kolkata, India) have authored the eleventh paper, whose title is: "Identifying Hazardousness of Sewer-Pipeline Gas-Mixture using Classification Methods: A Comparative Study." The primary goal of this work is to identify the level of hazardousness of sewer pipeline to offer safe and nonhazardous access to sewer-pipeline workers. Several classification algorithms are used, and their performances are evaluated and compared, both empirically and statistically, over the available data sets. Moreover, the performances of several ensemble methods are analyzed to understand the extent of improvement offered by these methods. The results have shown that the instance-based learning algorithm performed better than many others such as multilayer perceptron, radial basis function network, support vector machine and reduced pruning tree.

The twelfth paper is entitled "Efficient Surrogate Method for Predicting Pavement Response to Various Tire Configurations" and it is written by Mojtaba Ziyadi and Imad L. Al-Qadi (University of Illinois at Urbana-Champaign, USA) It is an efficient engineering application of ANN. More specifically, a computationally efficient surrogate model is developed based on Artificial Neural Networks to investigate the effect of the new generation of wide-base tires on pavement responses. Non-uniform tire contact stress measurements were obtained using a stressin-motion instrument. The measured 3-D contact stresses were applied on two extreme 3-D flexible pavement finiteelement models representing low-volume (thin) and highvolume (thick) roads. Eleven critical pavement responses were modeled at two different material properties input levels detailed and simplified depending on data availability.

Ebha Koley, Khushaboo Verma and Subhojit Ghosh (National Institute of Technology, Raipur, India) have authored the thirteenth paper "A modular neuro-wavelet based non unit protection scheme for zone identification and fault location in six phase transmission line." Sixphase transmission lines are proposed as a potential alternative to increase the power transfer capability of existing transmission lines without major modification in the existing structure of three-phase double-circuit system. This paper proposes a protection scheme for six-phase transmission line based on the hybridization of discrete wavelet transform and modular ANN. The fault information (approximate coefficients) in the voltage and current signals is captured using discrete wavelet transform. The standard deviation of the coefficients of voltage and current signals in each phase is then computed and given as input to modular ANN, which aims at identifying the faulty section/zone and estimate its location.

We do hope that this special issue covering multidisciplinary domains will inspire further innovative applications of neural computing, in both supervised and unsupervised manner. Computational intelligence can produce robust models that can be used as invaluable tools, to improve quality of life by offering more efficient engineering. 